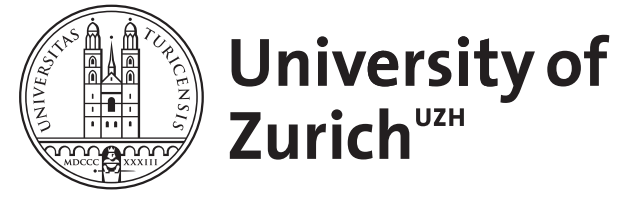
Archive

University of Zurich

University Library

Strickhofstrasse 39

CH-8057 Zurich

www.zora.uzh.ch

Year: 2015

\title{
New frontiers in neurooncology
}

Preusser, M ; Roth, P

DOI: https://doi.org/10.1097/WCO.0000000000000259

Posted at the Zurich Open Repository and Archive, University of Zurich

ZORA URL: https://doi.org/10.5167/uzh-115214

Journal Article

Accepted Version

Originally published at:

Preusser, M; Roth, P (2015). New frontiers in neurooncology. Current Opinion in Neurology, 28(6):626627.

DOI: https://doi.org/10.1097/WCO.0000000000000259 
Editorial for Current Opinion in Neurology, Neoplasms issue 2015

\section{New frontiers in Neurooncology}

Matthias Preusser (1), Patrick Roth (2)

(1) Department of Medicine I, Comprehensive Cancer Center CNS Unit (CCC-CNS), Medical University of Vienna

(2) Department of Neurology and Brain Tumor Center, University Hospital Zurich and University of Zurich

\section{Correspondence to:}

Matthias Preusser, MD

Department of Medinice I

Comprehensive Cancer Center CNS Unit (CCC-CNS)

Medical University of Vienna

Waehringer Guertel 18-20

1090 Vienna

Austria

Tel: 004314040044570

Email: matthias.preusser@meduniwien.ac.at

Patrick Roth, MD

University Hospital Zurich

Department of Neurology

Frauenklinikstrasse 26

$\mathrm{CH}-8091$ Zurich

phone: +41 (0)44 2555511

fax: +41 (0)44255 4507

email: patrick.roth@usz.ch 


\section{Main text}

Brain tumors are highly heterogeneous neoplasms and show a marked variability in their biology and clinical presentation. Over the past decades brain tumor research has evolved and today is one of the most dynamic scientific areas in oncology. In this issue of Current Opinion in Neurology, some of the most innovative, but at the same time controversial issues in contemporary neurooncology will be discussed. The topics reviewed include recent advances in tissue-based classification of brain tumors, the emerging role of immunotherapies in brain tumors, the significance of chemotherapy for the treatment of low-grade gliomas and the potential value of tumor-treating fields as a novel treatment modality of glioblastoma. Although covering various aspects of neurooncology, one common denominator of these topics is that they are controversially debated in the international community and will likely continue to fuel ongoing discussions in the next years.

The classification of tumors of the nervous system has so far been based on histopathological tumor appearance and the most commonly used classification system is regularly published and updated by the World Health Organization (WHO) [1]. However, insights from the last years have demonstrated that molecular information significantly contributes to better prognostication of the clinical course of some brain tumors than histology alone. For example, diffuse gliomas with or without mutation in one of the IDH genes have considerably different median overall survival times and molecular data such as the $1 p / 19 q$ status strongly correlate with sensitivity to cytotoxic therapies. Drs. Peter Wesseling and Elisabeth Jane Rushing summarize current discussions focusing on how to incorporate these molecular markers into the classical histopathological diagnoses to provide a clear and clinically useful modern classification system for brain tumors.

Low-grade gliomas are rare brain tumors and for decades the treatment of these tumors was limited by the lack of clinical trial data as a basis for evidence-based decision-making [2]. Recently however, data from adequately powered clinical studies performed over long periods of time and through effective international collaboration became available. The results obtained from these studies answered some questions, but gave raise to new questions as well as challenges and Dr. van den Bent will discuss the issues arising with regard to optimal selection and timing of chemotherapy for patients with low-grade gliomas. 
The treatment of glioblastoma is challenging and despite a wealth of pathobiological and clinical studies conducted over the past decades, the prognosis of glioblastoma patients is still dismal with overall survival times of approximately 15-17 months within clinical trials despite multimodal therapy [3]. Unfortunately, a number of rationally targeted therapeutic interventions such as drugs targeting specific molecular aberrations found in glioblastoma have not been successful. Therefore, there is a strong need to identify novel and effective treatment approaches for this devastating disease. Recent findings suggest that novel immunotherapeutic approaches such as advanced vaccination strategies or immune checkpoint modulators as well as the application of electromagnetic fields may provide some benefit for glioblastoma patients. The reviews by Drs. Weiss, Weller \& Roth, Bienkowski \& Preusser and Chamberlain \& XXX in this issue of Current Opinion in Neurology aim at providing a balanced overview of the available data and the current controversies in these dynamic fields of research.

We are confident that the topical reviews in this issue will help to catalyze the most relevant critical issues from the currently available evidence that need to be addressed by further studies. After all, only well-designed and strictly controlled investigations tailored to answer specific uncertainties will allow us to unequivocally answer open questions and make advances that truly benefit our patients. 


\section{References:}

1. Louis DN, Ohgaki H Wiestler OD, Cavenee WK (Editors). WHO Classification of Tumours of the Central Nervous System, $4^{\text {th }}$ Edition, Lyon: IARC Press; 2007.

2. Soffietti R, Baumert BG, von Deimling A, et al. Guidelines on management of low-grade gliomas: report of an EFNS-EANO Task Force. Eur J Neurol 2010; 17:1124-33

3. Weller M, van den Bent MJ, Hopkins K, et al. EANO guideline for the diagnosis and treatment of anaplastic gliomas and glioblastoma. Lancet Oncol 2014; 15:e395-403 